\title{
Adesão aos indicadores de segurança do paciente na assistência em saúde em um hospital escola
}

RESUMO | Objetivo: identificar a adesão dos profissionais de um hospital escola, quanto a utilização dos indicadores de segurança do paciente preconizados pelo Ministério da Saúde. Método: estudo descritivo exploratório, retrospectivo, de abordagem quantitativa, foi avaliado a adesão aos indicadores de segurança do paciente no período de outubro de 2019 a março de 2020. A coleta de dados foi no mês de março de 2020, no Núcleo de Segurança do Paciente e Gestão da Qualidade, por meio de dados coletados mensalmente pelo setor. Resultados: A taxa média de adesão à pulseira de identificação foi 81,9\%; identificação do paciente, taxa média de 61,7\%; média de adesão à escala de Braden de 80,6\% e a taxa de adesão a Escala de Morse, de 78,5\%. Conclusão: o não cumprimento dos protocolos instituídos pela instituição relativa à segurança do paciente expõe os usuários a eventos adversos preveníeis e compromete a qualidade da assistência em saúde.

Palavras-chaves: Segurança do paciente; Eventos adversos; Lesão por pressão.

\begin{abstract}
Objective: to identify the adherence of professionals from a teaching hospital, regarding the use of patient safety indicators recommended by the Ministry of Health. Method: exploratory, retrospective descriptive study, with a quantitative approach, adherence to patient safety indicators in the period from October 2019 to March 2020. Data collection took place in March 2020, at the Patient Safety and Quality Management Center, through data collected monthly by the sector. Results: The average rate of adherence to the identification bracelet was $81,9 \%$; patient identification, mean rate of $61,7 \%$; average adherence to the Braden scale of $80,6 \%$ and the adherence rate to the Morse scale, $78,5 \%$. Conclusion: failure to comply with the protocols established by the institution regarding patient safety exposes users to preventable adverse events and compromises the quality of health care.
\end{abstract}

Keywords: Patient safety; Adverse events; Pressure injury.

RESUMEN | Objetivo: identificar la adherencia de los profesionales de un hospital universitario, respecto al uso de los indicadores de seguridad del paciente recomendados por el Ministerio de Salud. Método: estudio descriptivo, exploratorio, retrospectivo con abordaje cuantitativo, se evaluó la adherencia a los indicadores de seguridad del paciente en el período de octubre de 2019 a marzo de 2020. La recogida de datos tuvo lugar en marzo de 2020, en el Centro Seguridad del Paciente y Gestión de Calidad, utilizando datos recogidos mensualmente por el sector. Resultados: La tasa promedio de adherencia al brazalete de identificación fue del 81,9\%; identificación de pacientes, tasa promedio del 61,7\%; adherencia media a la Escala de Braden del 80,6\% y la tasa de adherencia a la escala Morse del 78,5\%. Conclusión: el incumplimiento de los protocolos establecidos por la institución relativos a la seguridad del paciente expone a los usuarios a eventos adversos prevenibles y compromete la calidad de la atención en salud. Palabras claves: Seguridad del paciente; Eventos adversos; Lesión por presión.

\section{Ana Cláudia Ramos de Paula}

Enfermeira. Residente do Programa de Residência em Enfermagem na Especialidade de Gerenciamento de Enfermagem em Clínica Médica e Cirúrgica da Universidade Estadual do Oeste do Paraná, campus de Cascavel/PR. ORCID: 0000-0002-4704-7694

\section{Nelsi Salete Tonini}

Enfermeira. Doutora em Enfermagem pela Universidade de São Paulo/USP. Docente do Programa de Residência em Enfermagem na Especialidade de Gerenciamento de Enfermagem em Clínica Médica e Cirúrgica da Universidade Estadual do Oeste do Paraná, campus de Cascavel/PR.

ORCID: /0000-0003-4704-7634

\section{Maristela Salete Maraschin}

Enfermeira. Mestre pela Universidade Federal de Santa Catarina/UFSC. Docente do
Programa de Residência em Enfermagem na Especialidade de Gerenciamento de Enfermagem em Clínica Médica e Cirúrgica da Universidade Estadual do Oeste do Paraná, campus de Cascavel/PR.

ORCID: 0000-0003-2184-5056

\section{Daniele Lopes}

Enfermeira Especialista em Gerenciamento de Enfermagem em Clínica Médica e Cirúrgica. Enfermeira Coordenadora do Núcleo de Segurança e Gestão da Qualidade do Hospital Universitário do Oeste do Paraná. Cascavel/PR.

ORCID: 0000-0002-7666-2567

Recebido em: 13/05/2021

Aprovado em: 15/05/2021
INTRODUÇÃO

$\Lambda$ avaliação dos serviços de saúde e da assistência por meios de indicadores é imprescindível como elemento do cotidiano de trabalho em saúde, de modo a permitir a identificação de fragilidades e a visualização de oportunidades de melhorias. ${ }^{1}$ Nessa perspectiva, as ações de cuidado da equipe de saúde precisam ser monitorizadas, buscando conhecer seus resultados e estabelecer boas práticas com base em evidências.

Os indicadores de qualidade podem ser um meio de mensurar e avaliar as ações dos profissionais de saúde, são considerados instrumentos de gestão que orientam o caminho para a excelência do cuidado, 
eles se constituem na maneira pela qual os profissionais de saúde verificam uma atividade, monitoram aspectos relacionados à determinada realidade e avaliam o que acontece com os pacientes, apontando a eficiência e eficácia de processos e os resultados organizacionais. ${ }^{1,2}$

A segurança do paciente hospitalizado teve seus primórdios com Florence Nightingale ainda no século XIX, sua preocupação com o ambiente hospitalar e o meio ambiente já em 1863 enfatizava a comodidade do paciente com as condições locais, como por exemplo, a iluminação, a limpeza, o sanitarismo, a ventilação, a temperatura, a atenção, o cuidado, os odores e os ruídos. Em seu intenso cuidado, ela priorizava o isolamento, uma dieta adequada, a individualização do cuidado, e a redução do número de leitos por enfermaria, evitando, dessa forma, contaminações cruzadas e diminuindo a circulação de pessoas fora do serviço em âmbito hospitalar, prevenindo condições desfavoráveis aos pacientes. ${ }^{5}$

Em 2001, a Agência Nacional de Vigilância Sanitária (Anvisa) criou a Rede Brasileira de Hospitais Sentinelas, uma iniciativa Nacional para a segurança do paciente, que tinha como objetivo a identificação e notificação de problemas, sua função era observar e notificar desvios de qualidade em medicamentos (farmacovigilância), equipamentos (tecnovigilância) e sangue/hemoderivados (hemovigilância). Os registros dos problemas deveriam ser feitos no Sistema Nacional de Notificações para a Vigilância Sanitária (NOTIVISA), atualmente 193 instituições fazem parte da Rede Sentinela visando melhorar a segurança e assistência à saúde. ${ }^{6}$

Internacionalmente, nos últimos anos, a utilização dos indicadores para mensurar o desempenho e a atuação hospitalar tornou-se prática padrão. ${ }^{3,4} \mathrm{Um}$ estudo realizado na Holanda, constatou associação estatística entre o processo de prevenção de Lesão Por Pressão e a ocorrência de lesão de pele, evidenciando que sua prevalência está relacionada à qualidade do atendimento; portanto, o monitoramento desse indicador de processo poderá fornecer informações para mudanças futuras. ${ }^{3}$

Nesse contexto, o tema segurança do paciente vem sendo difundido e aprimorado nos cenários dos serviços de saúde, devido à importância em assegurar a qualidade dos serviços prestados aos pacientes. Um meio de verificar a qualidade da assistência é a avaliação de indicadores (índices estabelecidos como seguros nas instituições) relacionados à assistência.

Em 2013, o Ministério da Saúde instituiu o Programa Nacional de Segurança do Paciente (PNSP) que promoveu seis protocolos imprescindíveis a todos os serviços de saúde, referentes à segurança do paciente, sendo eles: 1 - Identificação do paciente; 2- Comunicação entre profissionais de saúde; 3- Uso de medicamentos (prescrição/dispensação/administração); 4- Cirurgia Segura; 5- Higiene das mãos e 6- Prevenção de quedas e lesão por pressão.

Dentre esses protocolos, discutiremos sobre o protocolo de identificação do paciente e o protocolo de quedas e lesão por pressão. O Protocolo de Identificação do Paciente tem como objetivo garantir a identificação correta do paciente e reduzir a ocorrência de incidentes que possam Ihe causar danos, todos os pacientes devem estar com dois tipos de identificação correta nas instituições hospitalares, desde a admissão até ao momento da alta. ${ }^{7}$

O Protocolo de Prevenção de Quedas tem como finalidade reduzir a ocorrência de quedas em pacientes hospitalizados e consequentemente reduzir o dano decorrente da queda. Um meio de redução é a implementação de medidas que avaliem o risco do paciente para a queda. ${ }^{7}$

A Escala de Morse "Morse Fall Scale" é a escala mais utilizada nas unidades hospitalares brasileiras, na versão traduzida e validada para o português.8 Possuí 6 itens a serem avaliados: histórico de quedas, diagnóstico secundário, auxílio na deambulação, terapia endovenosa/dispositivo endovenoso salinizado ou heparinizado, marcha e estado mental. Podendo pontuar de 0 a 125, sendo 0-24 risco baixo; 25-44 risco moderado e $\geq 45$ riscos alto. É aplicada na admissão do paciente e a cada $24 \mathrm{~h}$, sendo específica para pacientes com mais de 18 anos de idade, contudo não deverá ser aplicada em pacientes sedados, tetraplégicos e/ou com diagnóstico de morte encefálica. ${ }^{9}$

O Protocolo de Prevenção de Lesão por Pressão tem como objetivo promover a prevenção da ocorrência de Lesão Por Pressão (LPP) e outras lesões da pele, decorrentes dos longos períodos de internação ou fatores de riscos, como idade avançada e restrição ao leito. Para avaliar o risco de LPP, utiliza-se a escala Preditiva de Braden, que permite a avaliação de seis fatores de risco: percepção sensorial, atividade, mobilidade, umidade, nutrição, fricção ou cisalhamento. Essas escalas são pontuadas de um a quatro, exceto fricção ou cisalhamento, cuja medida varia de um a três. $\mathrm{O}$ escore total pode variar de 6 a 23 pontos, sendo os pacientes classificados da seguinte forma: risco muito alto (escores $\leq 9$ ), risco alto (escores de 15 a 18 pontos) e sem risco (escores $\geq 19$ ). ${ }^{10}$

A adesão a esses protocolos é mensurada por meio da análise de indicadores e é imprescindível nos serviços de saúde, haja vista que permite a gestão dos serviços de saúde, e, de acordo com os resultados encontrados, tomar decisões que venham interferir positivamente na assistência. Sua função é indicar falhas e problemas e em contrapartida apontar as sugestões e soluções, com foco educacional. ${ }^{6}$

Por meio da análise e levantamento dos indicadores, espera-se atingir a qualidade do conjunto das atividades desenvolvidas. Quando os indicadores assistenciais apontarem falhas no resultado, deve ser investigado o processo de trabalho.

Ante o exposto, questionou-se: se ocorre a adesão dos profissionais de um hospital escola, quanto a utilização dos indicadores de segurança do paciente? Para tanto, este estudo teve como objetivo identificar a adesão dos profissionais de um hospital escola, quanto a utilização dos indicadores de segurança do paciente preconizados pelo Ministério da Saúde. 
MÉTODO

Trata-se de um estudo descritivo exploratório, retrospectivo, de abordagem quantitativa realizado em um Hospital Universitário localizado no Oeste do Paraná. O referido Hospital atende exclusivamente pelo Sistema Único de Saúde, sendo referência em inúmeras especialidades, entre elas, traumas, atendendo pacientes de média e alta complexidade. Possui 238 leitos, subdivididos entre especialidades, incluindo Pronto Socorro/Sala de Emergência, UTIs e Enfermarias, o estudo compreende o perído de outubro de 2019 a março de 2020. Desses 238 leitos, no momento da avaliação mensal, o número de pacientes hospitalizados oscilou, sendo assim o " $\mathrm{n}$ " referente ao cálculo da porcentagem de adesão varia em cada mês (outubro: n 160, novembro n 158, dezembro n 168, janeiro n 176, fevereiro n 170 , março $n$ 185).

Tabela 1 - Adesão ao protocolo de Identificação do paciente, quanto ao uso de pulseira e identificação do leito. HUOP - Cascavel, 2020.

\begin{tabular}{|c|c|c|c|}
\hline Mês: & $\mathrm{N}^{\circ}$ de pacientes avaliados & Adesão/Pulseira: & Adesão/Leito (quadro) \\
\hline Outubro 2019 & n 160 & n $140,8-(88 \%)$ & n $88-(55 \%)$ \\
\hline Novembro 2019 & n 158 & n $126,4-(80 \%)$ & n $90-(57 \%)$ \\
\hline Dezembro 2019 & n 168 & n $136-(81 \%)$ & n $97,4-(58 \%)$ \\
\hline Janeiro 2020 & n 176 & n $140,8-(80 \%)$ & n $123,2-(70 \%)$ \\
\hline Fevereiro 2020 & n 170 & n $129,7-(76,3 \%)$ & n $117,3-(69 \%)$ \\
\hline Março 2020 & n 185 & n $159,4-(86,2 \%)$ & n $113,3-(61,2 \%)$ \\
\hline Média em 6 meses: & n 169,5 & n $138,8-(81,9 \%)$ & n $104,5-(61,7 \%)$ \\
\hline
\end{tabular}

Fonte: Núcleo de gestão da qualidade - HUOP, 2020.

Tabela 2 - Taxa de Adesão as Escalas de Braden e Morse. HUOP - Cascavel, 2020.

\begin{tabular}{|c|c|c|c|}
\hline Mês: & $\begin{array}{l}\mathrm{N}^{\circ} \text { de pacientes ava- } \\
\text { liados }\end{array}$ & $\begin{array}{c}\text { Adesão - Escala } \\
\text { de Braden }\end{array}$ & $\begin{array}{c}\text { Adesão - Escala de } \\
\text { Morse }\end{array}$ \\
\hline Outubro 2019 & n 122 & n $102,4-(84 \%)$ & n $103,7-(85 \%)$ \\
\hline Novembro 2019 & n 134 & n $116,5-(87 \%)$ & n $109,8-(82 \%)$ \\
\hline Dezembro 2019 & n 142 & n $120,7-(85 \%)$ & n $124,9-(88 \%)$ \\
\hline Janeiro 2020 & n 125 & n 93,7 - (75\%) & n $82,5-(66 \%)$ \\
\hline Fevereiro 2020 & n 125 & n 93,7-(75\%) & n $93,7-(75 \%)$ \\
\hline Março 2020 & n 132 & n 101,4-(78\%) & n $99-(75 \%)$ \\
\hline Média em 6 meses: & n 130 & n $104,7-(80,6 \%)$ & n $102-(78,5 \%)$ \\
\hline
\end{tabular}

Fonte: Núcleo de gestão da qualidade - HUOP, 2020.
Os indicadores avaliados no estudo formas preconizadas pelo Protocolo: Pulseira e Leito; Escala de Braden e Escala de Morse, posteriormente, os dados eram tabulados em uma planilha própria do Núcleo de Segurança do Paciente e Gestão da Qualidade do hospital estudado.

A coleta dos dados ocorreu no período de março a abril de 2020, por meio da análise da planilha no qual os dados são tabulados no Núcleo de Segurança do Paciente e Gestão da Qualidade do hospital estudado.

A coleta de dados pelo Núcleo de Sebusca ativa no prontuário eletrônico dos pacientes e observação in loco. No prontuário eletrônico, é verificada a adesão as Escalas de Braden e Morse, realizadas pelos enfermeiros das unidades. Na observação in loco é verificado leito a leito a pulseira e o quadro de identificação, por foram: Identificação do paciente por duas gurança do Paciente, se dá por meio de vezes questionado ao paciente e acompanhante a confirmação, não podendo exceder 24 horas da avaliação registrada pelos enfermeiros.

$\mathrm{Na}$ Instituição, para as avaliações, os pacientes são divididos em turnos de acordo com o horário em que são encaminhados para o banho, a fim de facilitar as avaliações em escalas e conferências das identificações pelos enfermeiros.

Os dados obtidos foram sumarizados em planilhas eletrônicas do software Microsoft Office Excel@ versão 2007 e, posteriormente, analisados por estatística descritiva, obtida pelas medidas de frequência relativa e absolutas dos casos notificados e posteriormente analisados e dispostos em tabela.

Os critérios de exclusão seguindo o Protocolo, foram: para a Escala de Braden crianças menores de um ano de idade e para a Escala de Morse pacientes menores de 18 anos de idade e pacientes sedados.

Consideraram-se os preceitos éticos referentes à pesquisa que envolve seres humanos estabelecidos pela Resolução $N^{\circ}$ 466/12 do Conselho Nacional de Saúde. A pesquisa foi avaliada pelo Comitê Permanente de Ética em Pesquisa Envolvendo Seres Humanos da Universidade Estadual do Oeste do Paraná. ${ }^{11}$

A pesquisa está inserida em um projeto maior, intitulado "Construção de indicadores assistenciais e gerenciais do Serviço de Enfermagem no Hospital Universitário do Oeste do Paraná/HUOP", aprovada conforme parecer $n^{\circ}$ 3.323.244 de 13 de maio de 2019.

\section{RESULTADOS}

Serão apresentados em tabelas os dados coletados e as taxas de adesões à Identificação do paciente com o uso de pulseira; Identificação no leito do paciente; Escala de Braden e Escala de Morse.

Em relação à Identificação do paciente nos seis meses de estudo, conforme a tabela 1, a média de pacientes avaliados ao mês foi 169,5 , sendo que a taxa média de adesão à pulseira de identificação 
foi $81,9 \%$. No mês de fevereiro, a taxa de adesão foi inferior à média dos demais meses, observa-se que a maior adesão ocorreu no mês de outubro (88\%). A Identificação do paciente no leito, que é realizada no quadro de identificação do paciente, teve a taxa de adesão média de $61,7 \%$ nos seis meses.

Em relação aos pacientes avaliados, a média de adesão à escala de Braden no período do estudo foi de $80,6 \%$, sendo que os meses de janeiro, fevereiro e março de 2020 têm adesão à escala inferior à média. A taxa de adesão a Escala de Morse, teve média em seis meses de 78,5\%, sendo que os meses de janeiro, fevereiro e março, assim como a Escala de Braden, também tiveram adesão inferior à média do período do estudo na Instituição.

\section{DISCUSSÃO}

A identificação do paciente, referida na Tabela 1, faz parte do protocolo de segurança do paciente estabelecido pelo Ministério da Saúde. Esse protocolo tem por objetivo identificar o paciente por duas formas, a fim de reduzir ao mínimo aceitável a ocorrência de eventos adversos relacionados ao pacientes. ${ }^{7}$

A Instituição estudada adotou a pulseira branca de identificação que contém os seguintes dados: nome do paciente, nome da mãe e data de nascimento e o quadro de identificação a beira leito que contém os demográficos do paciente, como nome, número do prontuário, nome do médico, riscos que o paciente apresenta (alergia, flebite, queda, bronco aspiração), data em que foi realizada a avaliação e nome do enfermeiro que realizou a avaliação.

A avaliação da adesão dos profissionais a identificação dos pacientes é fundamental para conscientização da importância desse protocolo, haja vista que a identificação correta previne danos que podem ser irreversíveis aos pacientes.

No momento da avaliação, verifica-se então se esses dados estão completos, e a data de avaliação deve ser referente às últimas 48h. Foram considerados com
Em relação aos pacientes avaliados, a média de adesão à escala de Braden no período do estudo foi de $80,6 \%$, sendo que os meses de janeiro, fevereiro e março de 2020 têm adesão à escala inferior à média. A taxa de adesão a Escala de Morse, teve média em seis meses de $78,5 \%$, sendo que os meses de janeiro, fevereiro e março, assim como a Escala de Braden, também tiveram adesão inferior à média do período do estudo na Instituição. identificação completa os quadros que atendiam a todos os quesitos supracitados, quadros parcialmente incompletos não são aceitos e são classificados como não identificados.

No que se refere à identificação do paciente, os itens pulseira e quadro, apresentados nas Tabelas 1, não tiveram médias mensais superiores a $90 \%$, quando o ideal preconizado é $100 \%$ de adesão aos indicadores de segurança. Segundo Souza, as instituições brasileiras possuem fragilidades relacionadas à cultura de segurança, evidenciadas pela baixa adesão aos treinamentos e a comunicação deficiente, determinantes para a não adesão integral aos protocolos da Organização Mundial da Saúde (OMS). ${ }^{12}$

A prevenção das lesões por pressão também faz parte dos protocolos de segurança do paciente estabelecidos pelo $\mathrm{Mi}$ nistério da Saúde. Dentro desse protocolo, a Escala de Braden é a mais utilizada para mensurar o risco do paciente em desenvolver lesão por pressão, seu uso associado aos cuidados preventivos prestados pela equipe de saúde, como mudança de decúbito frequente, por exemplo, podem apresentar diminuição da incidência de lesão. ${ }^{13}$ Conforme apresentado na Tabela 2 , a adesão média a essa escala no período do estudo é 80,6\%.

As escalas de avaliação de risco de desenvolvimento de lesão por pressão são importantes para os enfermeiros, haja vista que possibilitam identificar pontos vulneráveis, reforçar a avaliação constante e estimular a prevenção dessas lesões. ${ }^{10}$

Quanto à avaliação do risco de queda dos pacientes, também demonstrada na Tabela 2, a Escala de Morse é a escala mais utilizada nas unidades hospitalares brasileiras. ${ }^{9}$ Entretanto, observa-se que a média de adesão a essa escala no período de seis meses foi baixa, o que demonstra que os profissionais necessitam de constantes treinamentos para entenderem a importância da adesão a essa escala e medidas a serem tomadas após a avalição dos pacientes.

Após realizar a escala de Morse, os pacientes que apresentam risco de queda 
médio e alto, devem receber uma pulseira que identifica o risco de queda na Instituição (padronizada como amarela), o objetivo do uso da pulseira é que todos os profissionais que prestam cuidados ao paciente adotem condutas de prevenção a queda, como manter as grades do leito elevadas, auxiliar o paciente no momento da deambulação etc.

Para Souza, a não adesão aos protocolos de segurança do paciente é resultado da falta de treinamentos e de problemas relacionados à comunicação. ${ }^{12}$

\section{CONCLUSÃO}

A Segurança do Paciente é um tema atual e de grandes proporções, haja vista que impacta diretamente na qualidade da assistência prestada pelas instituições. Sendo assim, as equipes dos serviços de saúde devem estar envolvidas nessa temática constantemente e, por meio de estudos como este, espera-se divulgar o tema e promover avanços a assistência prestada pela enfermagem.

A equipe de enfermagem é fundamental no processo de Segurança do Paciente, haja vista que o enfermeiro além de ava- liar os itens do estudo, como identificação dos pacientes e as escalas, identifica pontos vulneráveis, realiza medidas preventivas quanto à segurança do paciente e intervenções quando necessário organizar o processo de trabalho.

Os achados deste estudo revelam que a adesão aos protocolos recomendados pela OMS e pesquisados por este estudo ainda apresentam uma adesão abaixo do $100 \%$. Podemos atribuir a esses resultados as fragilidades relacionadas à cultura de segurança do paciente, à capacitação profissional e ao pouco engajamento pessoal e coletivo, no que tange à comunicação na equipe de saúde para divulgação e incorporação de todos da prática assistencial segura e de qualidade.

Os resultados permitem, também, evidenciar a fragilidade dos processos de trabalho em relação à segurança dos usuários em atendimento nas unidades assistenciais do hospital estudado, caracterizado como serviços de alta complexidade. Em concordância ao estabelecido nos protocolos de segurança para os serviços de saúde no Brasil, percebe-se a necessidade da implementação desses protocolos, por meio de normas e rotinas institucionais, e de acordo com a realidade local. Tais medidas, quando sistematizadas, poderão contribuir para a redução de potenciais riscos e promover a segurança e a qualidade no processo de trabalho em saúde. Assim, é fundamental o engajamento de gestores e profissionais de saúde no reconhecimento desta demanda e na adoção de medidas institucionais recomendadas pelo Ministério da Saúde brasileiro e por organizações internacionais.

As taxas atuais de adesão a identificação dos pacientes e as escalas de Braden e Morse são frutos de treinamentos e do estímulo por meio das avaliações realizadas pelo Núcleo de Segurança do Paciente da Instituição, mas ainda há muito trabalho a ser realizado, as equipes necessitam de treinamento constante para entenderem a importância da necessidade de mudança na cultura de segurança do paciente.

Conclui-se que o não cumprimento dos protocolos instituídos pela instituição relativas à segurança do paciente expõe os usuários a eventos adversos preveníveis e demanda ações sistematizadas para observância das diretrizes governamentais e promoção da qualidade da assistência em saúde.

\section{Referências}

1. Januário GC, Lemos SMA, Friche AAL, ALVES CRL. Quality indicators in a newborn hearing screening service. Braz J Otorhinolaryngol [Internet]. 2015 [cited 2017 Mar 16], 81(3):255-63. Available from: http://www.scielo.br/pdf/ bjorl/v81n3/pt_1808-8694-bjorl-81-03-00255.pdf.

2. Gabriel CS, Melo MRAC, Rocha FLR, Bernardes A, MIGUELACIT, SILVA, MLPS. Use of performance indicators in the nursing service of a public hospital. Rev. Latino-Am Enfermagem [Internet]. 2011 [cited 2017 Mar 16]; 19(5). Available from: http://www.scielo.br/pdf/rlae/v19n5/pt_24.pdf

3. Van Dishoeck AM, Looman CWN, Steyerber EW, Halfens RJG, Mackenbach JP. Performance indicators: the association between the quality of preventive care and the prevalence of hospital-acquired skin lesions in adult hospital patients. J Adv Nurs [Internet]. 2016 [cited 2017 Mar 16];72(11):2818-30. Available from: https://www.researchgate.net/publication/303951673_Performance_indicators_the_association.

4. Van Dishoeck AM, Lingsma HF, Mackenbach JP, Steyerberg EW. Random variation and rankability of hospitals using outcome indicators. BMJ Qual Saf [Internet]. 2011 [cited 2017 Mar 16];20(10):869-74. Available from: http:// qualitysafety.bmj.com/content/20/10/869.long.

5. Martins DF, Benito LAO. Florence Nightingale e as suas contribuições para o controle das infecções hospitalares. Universitas: Ciências da Saúde. 2016 jul-dez. 2016; 14(2):153-166.

6. Prates CG, Stadñik CM. Segurança do Paciente, Gestão de Riscos e Controle de Infecções Hospitalares. 1.ed, Porto Alegre: Editora Moirá; 2017.
7. ANVISA Agência Nacional de Vigilância Sanitária. Anexo 02: Protocolo de Identificação do Paciente. 2013 jul. Disponível em:< file:///C:/Users/admin/ Downloads/protoc_segurancaPrescricaoUsoAdministracaoMedicamentos. pdf $>$ Acesso em: 19 nov. 2020.

8. Urbanetto JS, Creutzberg M, Franz et al. Morse fall scale: translation and transcultural adaptation for the portuguese language. Rev. esc. enferm. USP. [Internet] 2013 jun; 47(3). Disponível: http://dx.doi.org/10.1590/S0080623420130000300007

9. Barbosa P, Carvalho L, Cruz S. Escala de Quedas de Morse: Manual de Utilização. São Paulo: Editora Escola Superior de Enfermagem do Porto, 2015.

10. Silva ALM, Rached CDA, Liberal MMC. A utilização da escala de Braden como instrumento preditivo para prevenção de lesão por pressão. Rev. Direito, em Foco. 2019; (1):1-11.

11. Brasil Ministério da Saúde. Resolução N 466, de 12 de Dezembro de 2012. Aprova as normas regulamentadoras de pesquisas envolvendo seres humanos. Brasília, 2012. Disponível em: < http://conselho.saude.gov.br/resolucoes/2012/ Res0466.pdf>. Acesso em: 27 out. 2020.

12. Souza LO, Pinho MC, Machado LR, Jacques A. 0 uso de protocolos de segurança do paciente nas instituições hospitalares. Rev. Brasileira De Pesquisa Em Saúde/Brazilian Journal of Health Research. 2017; 19(1):70-77.

13. Vieira PA. Efetividade da implantação de protocolos de prevenção de lesões por pressão: revisão integrativa da literatura. Dissertação (Mestrado). São Carlos: Gestão da Clínica, Universidade Federal de São Carlos; 2019. 